INPLASY

PROTOCOL

To cite: Huang et al.

Association between body mass index and mortality in patients with COVID-19: a systematic review and metaanalysis. Inplasy protocol

2020120090. doi:

10.37766/inplasy2020.12.0090

Received: 17 December 2020

Published: 17 December 2020

Corresponding author:

Huei-Kai Huang

drhkhuang@gmail.com

Author Affiliation:

Hualien Tzu Chi Hospital, Buddhist Tzu Chi Medical

Foundation, Hualien, Taiwan

Support: None.

Review Stage at time of this submission: Data extraction.

Conflicts of interest:

None.

\section{Association between body mass index and mortality in patients with COVID-19: a systematic review and meta-analysis}

Huang, $\mathrm{H}^{1}$; Bukhari, K2; Hung, D3; Chang, RH${ }^{4}$; Peng, CC5; Tu, Y6.

Review question / Objective: We aim to conduct a systematic review and dose-response meta-analysis to explore the relationship between body mass index (BMI) and mortality in patients with COVID-19. P: Patients diagnosed with COVID-19; E: Obesity, overweight, and underweight; C: Ideal weight; O: Mortality.

Condition being studied: Coronavirus disease 2019 (COVID-19) has become pandemic and spread worldwide, causing considerable deaths. Identifying individuals at higher risk of developing critical illness and death was critical to prevent and manage this emerging infectious disease. Some studies have linked obesity to more severe illness and higher mortality in COVID-19 patients. However, the relationship between underweight and COVID-19 mortality was still inconclusive; meta-analyses summarizing the dose-response relationship, considering both the underweight and obese population, were lack.

INPLASY registration number: This protocol was registered with the International Platform of Registered Systematic Review and Meta-Analysis Protocols (INPLASY) on 17 December 2020 and was last updated on 17 December 2020 (registration number INPLASY2020120090).

\section{INTRODUCTION}

Review question / Objective: We aim to conduct a systematic review and doseresponse meta-analysis to explore the relationship between body mass index (BMI) and mortality in patients with COVID-19. P: Patients diagnosed with
COVID-19; E: Obesity, overweight, and underweight; C: Ideal weight; O: Mortality.

Condition being studied: Coronavirus disease 2019 (COVID-19) has become pandemic and spread worldwide, causing considerable deaths. Identifying individuals at higher risk of developing critical illness and death was critical to prevent and 
manage this emerging infectious disease. Some studies have linked obesity to more severe illness and higher mortality in COVID-19 patients. However, the relationship between underweight and COVID-19 mortality was still inconclusive; meta-analyses summarizing the doseresponse relationship, considering both the underweight and obese population, were lack.

\section{METHODS}

Search strategy: We search PubMed, Embase, Cochrane Library, Scopus, and Web of Science using the keywords of "COVID-19," "body mass index," "obesity," "overweight," and "underweight.".

Participant or population: Patients diagnosed with COVID-19.

Intervention: Obesity, overweight, and underweight.

Comparator: Ideal weight.

Study designs to be included: Observational studies.

Eligibility criteria: Original studies that have evaluated the association between BMI and mortality in patients with COVID-19.

Information sources: Electronic databases: PubMed, Embase, Cochrane Library, Scopus, and Web of Science.

Main outcome(s): COVID-19 related and allcause mortality.

Quality assessment / Risk of bias analysis: We use the Newcastle-Ottawa Scale for evaluating the study quality of cohort and case-control studies.

Strategy of data synthesis: We will synthesize the risk ratios or odds ratios obtained from original studies to calculate the pooled RRs in our meta-analysis. We will calculate the pooled RRs and their $95 \%$ confidence intervals (CIs) using the DerSimonian and Laird random effects model. We will also perform a two-stage random-effects dose-response metaanalysis using a restricted cubic splines model with three-knots.

Subgroup analysis: The subgroup analyses will be performed for study design, country, study population, age, sex, patient numbers, and BMI categories.

Sensibility analysis: A sensitivity analysis will be conducted by omitting each study individually to evaluate the influence of each study on the overall pooled estimate. Another sensitivity analysis will be conducted by only pooling studies that have specifically evaluated underweight patients.

Country(ies) involved: The U.S. and Taiwan.

Keywords: COVID-19; body mass index; obesity; overweight; underweight; mortality.

Contributions of each author:

Author 1 - Huei-Kai Huang.

Author 2 - Khulood Bukhari.

Author 3 - Duan-Pei Hung.

Author 4 - Rachel Huai-En Chang.

Author 5 - Carol Chiung-Hui Peng.

Author 6 - Yu-Kang Tu. 